\title{
Editorials
}

\section{Cardiac arrest resuscitation and its relevance to general practice}

\section{INTRODUCTION}

Patients in trouble seek out the help of their GP in many everyday situations. Occasionally such situations represent life-threatening emergencies. Indeed, a proportion of GPs have for many years voluntarily contributed to structured systems of community emergency care via organisations such as the British Association for Immediate Care (BASICS). However, for individual GPS, cardiac arrest in the community is the key emergency that is likely to be confronted at some stage and should be prepared for. In Europe, out-of-hospital cardiac arrest (OHCA) has a population incidence of 38-86 per 100000 persons peryear., ${ }^{1,2}$ EuReCa ONE la 2014 prospective study of OHCA registries across the EU 27) showed that only $10.3 \%$ of patients were discharged from hospital or survived for longer than 30 days following cardiopulmonary resuscitation (CPR), with wide variations between countries and regions. ${ }^{3}$ The International Committee on Resuscitation (ILCOR) reviews the evidence on a 5 - yearly basis to provide clear guidance for $\mathrm{OHCA}$ resuscitation. While early CPR and defibrillation remain the key evidence-based interventions, ${ }_{1}^{4}$ little attention is paid to the role of general practice in the extensive guidelines on systems for delivery of this care.

In contrast to this observation, international evidence confirms that GPs have considerable exposure to cardiac arrest resuscitation via their day-to-day activities..$^{5-9}$ This is unsurprising as GPs work in the community where OHCA occurs, and work with patients and populations who are known to be at increased risk of OHCA as a result of the multimorbid medical conditions that are now prevalent in the community. Why then is the role of OHCA resuscitation not highlighted as a key element of general

\section{for individual GPS, cardiac arrest in the community is the key emergency that is likely to be confronted at some stage and should be prepared for.}

practice? General practice has at times itself expressed scepticism about preparedness for OHCA care and more widely the involvement of GPS in resuscitation has attracted little attention. . $^{10,11}$ Infrequent exposure, lack of training, non-availability of automatic external defibrillators (AEDs) or other equipment, fear of making mistakes, and personal distress, are among the many barriers which can be identified by GPs. Each of these barriers can however be addressed and overcome, and the importance of the role must be more widely acknowledged.

Existing evidence demonstrates that GP involvement in OHCA resuscitation is associated with substantially higher rates of survival than are otherwise expected. ${ }^{7,9,10}$ National level research carried out by our group over a 10-year period in Ireland found that half of all general practices and twothirds of rural practices encountered at least one cardiac arrest resuscitation attempt. ${ }^{9}$ The overall survival rate was $17 \%$, far in excess of the national survival rate of less than $7 \%$, with most survivors experiencing good functional outcomes. Furthermore, when cardiac arrest occurred at the practice premises, the survival rate rose as high as $47 \%{ }^{9}$

\section{KEY GP COMPONENTS OF CARDIAC ARREST CARE}

Cardiac arrest occurs commonly within the communities which GPs serve and thus has relevance to the role, yet what structured

\section{"The most important interventions in OHCA learly CPR and defibrillation) are time critical. When a patient collapses in the surgery, during a house call or nearby, the preparation of the GP and practice staff to deliver these interventions can make the difference between life and death.}

contributions can GPs make? The most important interventions in OHCA learly CPR and defibrillation) are time critical. When a patient collapses in the surgery, during a house call, or nearby, the preparation of the GP and practice staff to deliver these interventions can make the difference between life and death. The key general practice components of OHCA care include:

- availability of an AED in every practice;

- basic life support training and certification for all staff;

- effective links with local ambulance and emergency medical services;

- advanced life support provision at practice level;

- local alerting/response systems for OHCA in the immediate area;

- encouraging CPR training and awareness among our patients;

- identification of high-risk groups;

- facilitating advanced care directives/do not attempt CPR orders where appropriate; and,

- research initiatives.

Policy, funding, training, communications systems, and research frameworks to address these goals are urgently needed. The RCGP describes general practice as the provision of continuing, comprehensive, coordinated, and person-centred care to patients in their communities, and notes that GPs carry out $90 \%$ of patient contacts in the health service. ${ }^{12}$ Cardiac arrest is an uncommon clinical presentation in primary care; however, the accessibility of general practice means that many GPs will encounter cardiac arrest and should attempt resuscitation. When OHCA care is provided by GPs to their own patients it can be informed by a rounded understanding of the individual patient, with consideration of their care preferences, comorbid conditions, and baseline functional independence. 


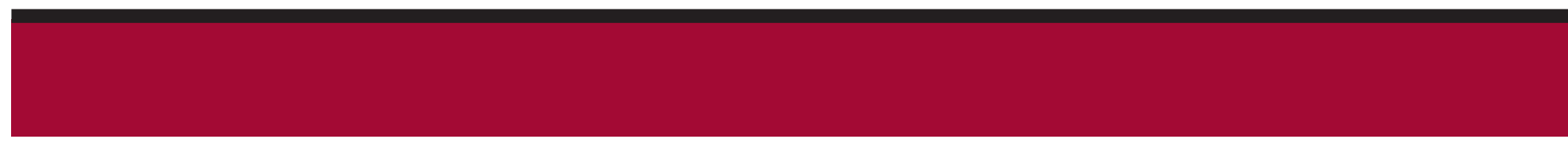

\section{"It is clear that when GPs are properly trained and equipped for cardiac arrest management, increased rates of survival can be achieved. When they are not, a vital opportunity is lost.}

Such understanding is likely to have relevance in terms of patient-centred, clinical, and ethical decision-making around continuing or ceasing resuscitation, and where appropriate, the instigation of compassionate end-of-life care.

When evaluated in terms of survival outcome, the existing evidence base confirms that GPs already make a significant contribution to OHCA resuscitation. The availability of a GP in the community affords the opportunity to provide fundamental care at an early stage following OHCA, and thus a greater chance of patient survival ultimately exists. It is clear that when GPs are properly trained and equipped for cardiac arrest management, increased rates of survival can be achieved. When they are not, a vital opportunity is lost. The literature suggests that not all GPs are aware of their potential in this critical area of practice, or are consistently equipped or trained to respond to OHCA. $8,10,13$ Further opportunity to save lives exists, and cardiac arrest management must be considered one of many priorities for day-to-day general practice. That process should begin within the specialist training and continuing medical education frameworks that underpin modern general practice, and should be supported by research on the clinical and health systems issues that can maximise the impact of GP led care.

In Ireland and the UK, GPs take on the care of individuals who suffer cardiac arrest within their communities, often without contractual reward and wholly in a voluntary capacity. ${ }^{14}$ This observation is at odds with a contemporary narrative of overwork, underpay, poor morale, and burnout, that is prevalent in the modern discourse of general practice. An alternative perspective offered by the work of these volunteer doctors is that the commitment to community, and the public service spirit that has led many doctors to become GPs, has just as important a place in the professional dialogue about general practice. In cardiac arrest care this commitment and spirit are underscored by an evidence base that confirms GP involvement in OHCA resuscitation can and does save lives.

\section{Tomás Barry,}

Lecturer and Assistant Professor, School of Medicine, University College Dublin, Dublin, Ireland.

\section{Gerard Bury,}

Professor of General Practice, School of Medicine, University College Dublin, Belfield, Dublin, Ireland.

\section{Provenance}

Freely submitted; externally peer reviewed.

\section{Competing interests}

The authors are GPs with roles in cardiac arrest education, research, and clinical care. The authors have declared no other competing interests.

DOI: https://doi.org/10.3399/bjgp19X705629

\section{ADDRESS FOR CORRESPONDENCE}

Tomás Barry

UCD Centre for Emergency Medical Science, School of Medicine, Health Sciences Centre, University College Dublin, Belfield, Dublin 4, Ireland.

\section{Email: tomas.barryaucd.ie}

\section{REFERENCES}

1. Berdowski J, Berg RA, Tijssen JG, Koster RW. Global incidences of out-of-hospital cardiac arrest and survival rates: systematic review of 67 prospective studies. Resuscitation 2010 . 81(11): 1479-1487.

2. Atwood C, Eisenberg MS, Herlitz J, Rea TD. Incidence of EMS-treated out-of-hospital cardiac arrest in Europe. Resuscitation 2005; 67(1): $75-80$.

3. Gräsner JT, Lefering R, Koster RW, et al. EuReCa ONE-27 Nations, ONE Europe, ONE Registry: a prospective one month analysis of out-of-hospital cardiac arrest outcomes in 27 countries in Europe. Resuscitation 2016; 105: 188-195

4. Perkins GD, Handley AJ, Koster RW, et al. European Resuscitation Council Guidelines for Resuscitation 2015. Section 2. Adult basic life support and automated externa defibrillation. Resuscitation 2015; 95: 81-99.

5. Ong ME, Chan $\mathrm{YH}$, Ang HY, et al. Resuscitation of out-of-hospital cardiac arrest by Asian primary health-care physicians. Resuscitation 2005; 65(2): 191-195.

6. Chalkias A, Koutsovasilis A, Mazarakis A, et al. Cardiac arrest in Greek primary health care and willingness of general practitioners to use automatic external defibrillator. Resuscitation 2011; 82(9): 1144-1147.

7. Colquhoun M. Resuscitation by primary care doctors. Resuscitation 2006; 70(2): 229-237.

8. Barry T, Bury G. Advisory external defibrillator availability in general practice. Ir Med J 2015; 108(7): 212-213.

9. Barry T, Headon M, Glynn R, et al. Ten years of cardiac arrest resuscitation in Irish general practice. Resuscitation 2018; 126: 43-48.

10. Colquhoun MC. Resuscitation in general practice - time for action: editorial on: 'The presence of resuscitation equipment and influencing factors at general practitioners' offices in Denmark: a cross-sectional study by Niegsch ML, Krarup NT, Clausen NE. Resuscitation 2014; 85(1): 5-6.

11. Toon PD. Do we spend too much time with Nellie the Elephant? Br J Gen Pract 2010; DOI: https://doi.org/10.3399/bjgp10X482167.

12. Royal College of General Practitioners. The National Health Service. 2019. https://nww. rcgp.org.uk/training-exams/discover-generalpractice/overseas-doctors-guide/the-nationalhealth-service.aspx (accessed 10 Sep 2019).

13. Soo L, Smith N, Gray D. The place of general practitioners in the management of outof-hospital cardiopulmonary resuscitation. Resuscitation 1999; 43(1): 57-63.

14. Barry T, Conroy N, Headon M, et al. The MERIT 3 project: alerting general practitioners to cardiac arrest in the community. Resuscitation 2017; 121: 141-146. 